\title{
Role of the pharmacist in improving inhaler technique and asthma management in rural areas in Jordan
}

This article was published in the following Dove Press journal:

Clinical Pharmacology: Advances and Applications

\author{
Iman A Basheti ${ }^{1}$ \\ Yara B Salhi' \\ Mariam M Basheti ${ }^{2}$ \\ Salim A Hamadi ${ }^{3}$ \\ Walid Al-Qerem ${ }^{4}$ \\ 'Department of Clinical Pharmacy and \\ Therapeutics, Faculty of Pharmacy, \\ Applied Science Private University, \\ Amman, Jordan; ${ }^{2}$ Faculty of Pharmacy, \\ Jordan University of Science and \\ Technology, Irbid, Jordan; ${ }^{3}$ Department of \\ Pharmacology \& Biomedical Sciences, \\ Faculty of Pharmacy and Medical \\ Sciences, Petra University, Amman, \\ Jordan; ${ }^{4}$ Department of Pharmacy, Al- \\ Zaytoonah University of Jordan, College \\ of Pharmacy, Amman, Jordan
}

Introduction: Pharmacists can have a valuable role in educating patients on correct inhaler technique leading to improved asthma management. Rural areas can benefit from the role of the pharmacist considering the barriers found in attending primary health-care facilities.

Objectives: This study aimed to assess the impact of inhaler technique education delivered by pharmacists on patients' inhaler technique, Asthma Control Test (ACT) score, forced expiratory volume in the first 1 second $\left(\mathrm{FEV}_{1} \%\right)$, and reliever use (puffs/day).

Methods: A pre-post interventional study was conducted over 6 months from February 2017 to July 2017 in rural areas in Jordan. Asthma patients visiting respiratory clinics and using metered dose inhaler (MDI) or turbuhaler (TH) controlled medication were randomly recruited. Inhaler technique was assessed via published checklists. The $\mathrm{ACT}, \mathrm{FEV}_{1} \%$, and reliever use (puffs/day) were assessed. Patients were educated on inhaler technique via demonstration with return demonstration education. All assessments were repeated 3 months post education.

Results: A total of 103 (TH, n=44; MDI, $\mathrm{n}=59$ ) patients were recruited (mean age $=46.5$ $\pm 13.5), 74 \%$ females. Patients reported an overuse of their reliever (5.1 \pm 4.2 puffs/day). Only 2 patients $(1.9 \%)$ had well-controlled asthma, while the rest had either moderately $(19.4 \%)$ or poorly $(78.6 \%)$ controlled asthma. Patients using the MDI achieved 3.03 \pm 4.30 ACT score improvement $(p<0.001)$, which is a clinically significant improvement in control. Patients using the $\mathrm{TH}$ achieved a statistically significant improvement of $2.07 \pm 4.72(p=0.031)$. $\mathrm{FEV}_{1} \%$ improved significantly for MDI users $(p=0.005)$ but not for TH users $(p=0.097)$. Reliever use decreased significantly for MDI and TH users.

Conclusion: Asthmatic patients living in rural areas in Jordan reported poor inhaler technique, ACT scores, and $\mathrm{FEV}_{1} \%$ scores and high use of reliever medications. Pharmacist-led educational intervention resulted in improved inhaler technique scores, $\mathrm{ACT}$ scores, and $\mathrm{FEV}_{1} \%$ scores and lowered reliever use over time.

Keywords: inhaler technique, asthma control, reliever use, rural area, pharmacist role

\section{Introduction}

Asthma is a serious global health-care issue, affecting an estimated 300 million individuals of all ages, with prevalence ranging from $1 \%$ to $18 \%$ in different countries. ${ }^{1}$ In lowresource countries, asthma prevalence has been increasing, ${ }^{1}$ while in Jordan there has been an alarming twofold increase in the prevalence of asthma over the past 10 years. ${ }^{2,3}$

The final goal of asthma treatment is to achieve optimal asthma control and to maintain this control thereafter. ${ }^{1}$ Many effective inhaled medications are found on the market, yet poor asthma control still exists with poor inhaler technique being one of the main determinants. ${ }^{4-6}$ Various formulations and designs of metered dose inhalers (MDIs) and dry powder inhalers (DPIs) are available; however, incorrect inhalation techniques
Correspondence: Iman A Basheti Applied Science Private University, P.O. Box 166, Amman, Jordan Tel +962799048003

Fax+96265515017

Email dr_iman@asu.edu.jo 
are still demonstrated by health-care professionals and their patients. ${ }^{5,7-9}$ MDIs are the most frequently used due to their familiarity, low cost, and the need for low inspiratory flow rates for adequate drug deposition in the patient's lung. ${ }^{10}$ DPIs, including the turbuhaler $(\mathrm{TH})$, are becoming more popular by the day, due to overcoming the coordination difficulty with using the MDIs, achieving greater drug deposition in the lung and lower side effects. ${ }^{11,12}$ In addition, combination medications in the one device have become a cornerstone in the management of asthma and this reflects current asthma management. ${ }^{1}$ However, DPI users have to inhale "as fast and as deep as they can" through their inhalers to achieve an effective fine particle dose with adequate lung deposition. ${ }^{13,14}$ Not all patients can equally achieve the required inhalation speed for effective therapeutic use. ${ }^{15,16}$ Both MDIs and DPIs require training and certain skills to achieve correct technique and deep deposition of the medication into the lungs. ${ }^{17-19}$

Not receiving proper asthma education or education about inhaler technique leads to uncontrolled disease. ${ }^{20}$ Inadequate asthma control is consequently translated into impairments in the patients' health-related quality of life. ${ }^{21}$

Rural areas in Jordan are areas not included in the central city and its surroundings; opposite to urban areas. ${ }^{22}$ Although the incidence of asthma is lower in rural areas than urban areas, ${ }^{23}$ asthma care provided by rural primary health-care teams lead to significant improvements in all indicators of quality asthma care provision to patients, although important barriers exist for rural practices to implement evidence-based asthma care practices. $^{24}$ In this perspective, pharmacists have a very vital role in providing both the initial training for first-time inhaler users and the subsequent regular, follow-up retraining. ${ }^{25-28}$

This study aimed to assess inhaler technique demonstration skills, asthma control through the Asthma Control Test (ACT), forced expiratory volume in the first 1 second $\left(\mathrm{FEV}_{1}\right)$, and reliever use (puffs/day) by asthma patients living in rural areas in Jordan. Secondly, the study aimed to assess the impact of inhaler technique education delivered by pharmacists on patients' inhaler technique, ACT scores, $\mathrm{FEV}_{1} \%$, and reliever use (puffs/day).

\section{Materials and methods}

A pre-post interventional study was conducted over 6 months from February 2017 to July 2017 in rural areas in Jordan. Ethics approval was obtained from the Jordanian Ministry of Health and from the hospitals at which the study was conducted.
Asthma patients from rural areas in Jordan visiting respiratory clinics in three public hospitals located in west Jordan (Al-Salt Hospital, Al-Salt), north Jordan (Princess Basma Hospital, Irbid), and middle of Jordan (Al Basheer Hospital, Amman) - were recruited into the study. Patients using a controlled medication by MDI or TH were randomly approached by the researcher and were invited to participate in the study. This study was conducted in accordance with the Declaration of Helsinki. Patients who agreed to participate following reading the information leaflet signed an informed consent form. A parent or legal guardian provided written informed consent for any participant under 18 years of age. Only asthma patients who met the following inclusion criteria were enrolled into the study: with a doctor diagnosis of asthma, above age 14 years, currently using inhaled corticosteroids with or without long-acting B2 agonists via TH or MDI, and have been on the same asthma inhaler for a minimum of 1 month prior to study enrollment. Patients were excluded if they did not self-administer their medication, or do not speak or understand Arabic.

Following recruitment, data were collected at baseline using a questionnaire designed to collect patients' demographics including age, gender, marital status, education, working status, smoking, and age of onset of asthma.

Information on asthma medications used, duration of inhaler use, frequency of usage, reliever use as number of puffs per day, number of canisters used per month, and any previous advice received on asthma inhaler use from any health-care provider was also collected.

A pilot study involving questionnaire completion by 30 asthma patients was carried out 1 month before the study to assess feasibility and to evaluate clarity and readability of the questionnaires. Results from this phase of the study were not included in the final analysis.

Asthma severity was assessed using the validated ACT in Arabic language. ${ }^{29}$ The ACT scores range from 5 to 25 (higher scores indicate better asthma control). The $\mathrm{ACT}^{30}$ is a validated questionnaire often used to evaluate asthma control in clinical care settings, ${ }^{31}$ reflecting the patient's status over the previous 4 weeks. The ACT was one of the tools recommended by the American Thoracic Society/ European RespiratorySociety Task Force on standardization of asthma control measures. ${ }^{32}$ Scores of 20-25 are classified as well-controlled asthma; 16-19 as not well-controlled asthma; and $5-15$ as very poorly controlled asthma. The ACT includes four symptom/reliever questions plus a patient self-assessed level of control. The minimum clinically important difference is 3 points. ${ }^{33}$ 
Spirometry provides an objective measure of lung obstruction, which adds to the reliability of lung assessment. Spirometry was performed using a spirometer (Spirolab; Medical International Research) with a disposable turbine (made in Italy). The patient's lung function, specifically $\mathrm{FEV}_{1} \%$, was collected and reported as a percentage. Patients were requested to perform three satisfactory blows to ensure that the documented forced expiratory volumes are "the patient's best". Patients used disposable mouthpieces.

Three months post baseline, all patients were contacted by the researcher and an appointment was made at the clinic at which they had their baseline assessment completed. During the second visit, patients were assessed on their inhaler technique, $\mathrm{ACT}$, and $\mathrm{FEV}_{1} \%$ by the same researcher who conducted the baseline assessment.

Inhaler technique was assessed by the researcher (baseline assessment) for the patient's controller device, using a placebo inhaler provided by AstraZeneca plc (London, UK) and GlaxoSmithKline plc (London, UK). The patient's TH and MDI inhaler technique was assessed by the researcher using validated and published inhaler technique checklists (Table 1). ${ }^{17,34}$ Patients were assessed on their correct technique, the correct performance of all steps in the checklist, and the correct essential technique, which is the correct demonstration of steps which if incorrectly performed mean little or no medication would reach the lung.

Patients were educated by the researcher using a specialized "Show and Tell" inhaler technique educational intervention to describe and demonstrate correct use, going through each step on the TH or MDI checklist (in Arabic). ${ }^{17,18,34}$

Patients were re-educated on the incorrect steps they performed or missed. This cycle of assessment and education was repeated up to 3 times if necessary, or until the patient demonstrated correct technique on all steps. The researcher used the inhaler technique checklist to highlight problem steps performed by the patient during assessment, explaining the importance of performing that step correctly. Verbal information, technique physical demonstration, and checklist written information were used. ${ }^{28}$

\section{Data analysis}

Data were analyzed using the Statistical Package for Social Science (SPSS) version 21 (IBM Corporation, Armonk, NY, USA). The primary outcome variable, change in inhaler technique score between baseline and 3 months, was assessed and tabulated for all patients. Differences with $p<0.05$ were considered statistically significant.
Table I Inhaler technique checklists

Step. Description/action
Turbuhaler Technique Checklist
1. Remove the cap from the inhaler
2. Keep inhaler upright ${ }^{\mathrm{a}}$
3. Rotate grip until a click is heard ${ }^{\mathrm{a}}$
4. Exhale to residual volume
5. Exhale away from mouthpiece
6. Place mouthpiece between teeth and lips
7. Inhale forcefully and deeply
8. Hold breath for 5 seconds
9. Exhale away from mouthpiece
Metered dose inhaler Technique Checklist
1. Remove mouthpiece cover and shake
2. Hold inhaler upright
3. Exhale to residual volume
4. Keep head upright or slightly tilted
5. Place mouthpiece between teeth and lips
6. Inhale slowly and press canister
7. Continue slow and deep inhalation
a
8. Hold breath for 5 seconds
9. Close the inhaler

Notes: These checklists and essential steps are in accordance to the literature. ${ }^{17,18,34}$ ${ }^{a}$ Essential step: if not performed correctly, little/no medication will reach the lung. ${ }^{16}$ 'This step is not included in the product insert but appears in the turbuhaler instructions on the Global Initiative for Asthma website,' and in the checklist from van der Palen et al. ${ }^{18}$

The proportions of patients who performed correct technique (all steps correct) and correct essential technique (essential steps correct) were compared using Pearson's $\chi^{2}$ test. For continuous variables, including inhaler technique scores, $\mathrm{ACT}$ scores, $\mathrm{FEV}_{1} \%$ scores, and reliever use (puffs/day), comparisons between groups at baseline and follow-up were performed by independent-sample $t$-test (or Mann-Whitney $U$-test for data that were not normally distributed) and Wilcoxon signed-rank test, respectively. Correlation analysis was performed using Pearson's correlation to identify relationships between inhaler technique scores, ACT scores, $\mathrm{FEV}_{1} \%$ scores, and reliever use (puffs/day).

In order to determine predictors of improved asthma symptom control over the study period, a multiple linear regression analysis was performed. The dependent variable was change in ACT score from baseline to follow-up visit. Independent variables included inhaler type (TH or $\mathrm{MDI}$ ), age, gender, and ACT scores at baseline and change in $\mathrm{FEV}_{1} \%$ scores and change in inhaler technique scores across the study. 


\section{Results}

A total of 103 asthmatic patients living in rural areas in Jordan were approached to participate in the study. All of the patients agreed to be enrolled in the study after signing the information consent form (Figure 1). Patients were using either a TH $(n=44)$ or an MDI $(n=59)$ for their controller treatment. Age of participants ranged between 14 and 79 years (mean age $=46.5 \pm 13.5$ ). The majority of participants were females (74\%), had elementary or high school education (85.5\%), were married $(77.7 \%)$, were unemployed (70.9\%), and were nonsmokers (66\%). More than $80 \%$ of patients developed their asthma after the age of 18 years (mean age $32.5 \pm 5.1$ ) and have been using their inhalers for over 12 years. No significant differences were found with regards to the demographic characteristics for TH and MDI users (Table 2). Patients reported an overuse of their reliever inhaler $\left(\right.$ Ventolin $\left.^{\circledR}\right)$, with an average of 5.1 $\pm 4.2 \mathrm{puffs} /$ day.

As for the ACT results, it was made evident that only 2 patients $(1.9 \%)$ had well-controlled asthma (ACT score $>19$ ), while the rest had either moderately controlled (ACT score $=16-19)(19.4 \%)$ or poorly controlled (ACT score $<16)$ asthma (78.6\%). The ACT mean score (out of 25 ) was very low (10.53 \pm 4.29$)$.

Patient education affected baseline ACT scores, as patients with elementary school education had lower ACT scores $(9.52 \pm 4.11)$ than those with higher school education $(11.29 \pm 5.38)$ or university degrees $(12.25 \pm 4.80)(p=0.038$, one-way ANOVA). Patients' age also affected baseline ACT scores, as higher age correlated with lower ACT scores $(\mathrm{r}=-0.238, p=0.015)$.

Patients had baseline $\mathrm{FEV}_{1} \% \leq 80 \%$ predicted $(54.36$ \pm 18.47 ), with a range from 16.0 to 80.0 for all patients.

As for inhaler technique assessment, the mean score (out of 9) was low (5.4 \pm 2.2$)$ for both inhaler users. Only 1 patient (TH user) performed correct inhaler technique. Similar results were found for correct essential technique (Table 3), with few patients demonstrating essential technique correctly for $\mathrm{TH}(6.8 \%)$ and $\mathrm{MDI}$ (7.3\%). All patients were able to demonstrate correct inhaler technique following education at baseline before leaving the respiratory clinics.

Almost all participants (95.15\%) had been prescribed their inhalers by a specialist (Table 4). All patients reported receiving a previous education on inhaler use, mostly via the hospital clinic $(83.50 \%)$. The advice was communicated verbally $(53.40 \%)$ for most people, and was provided mostly when patients were first prescribed their inhalers (70.87\%). Few patients (12.62\%) reported receiving counseling on inhaler technique during the previous 12 months, and only $6.80 \%$ had their inhaler technique checked following their first inhaler use. Significantly more patients using the TH $(p=0.022)$ believed they had correct technique $(47.73 \%)$ than patients using the MDI (37.29\%). More MDI users were not sure of their technique (72.88\%) compared to $\mathrm{TH}$ users $(25.00 \%)$.

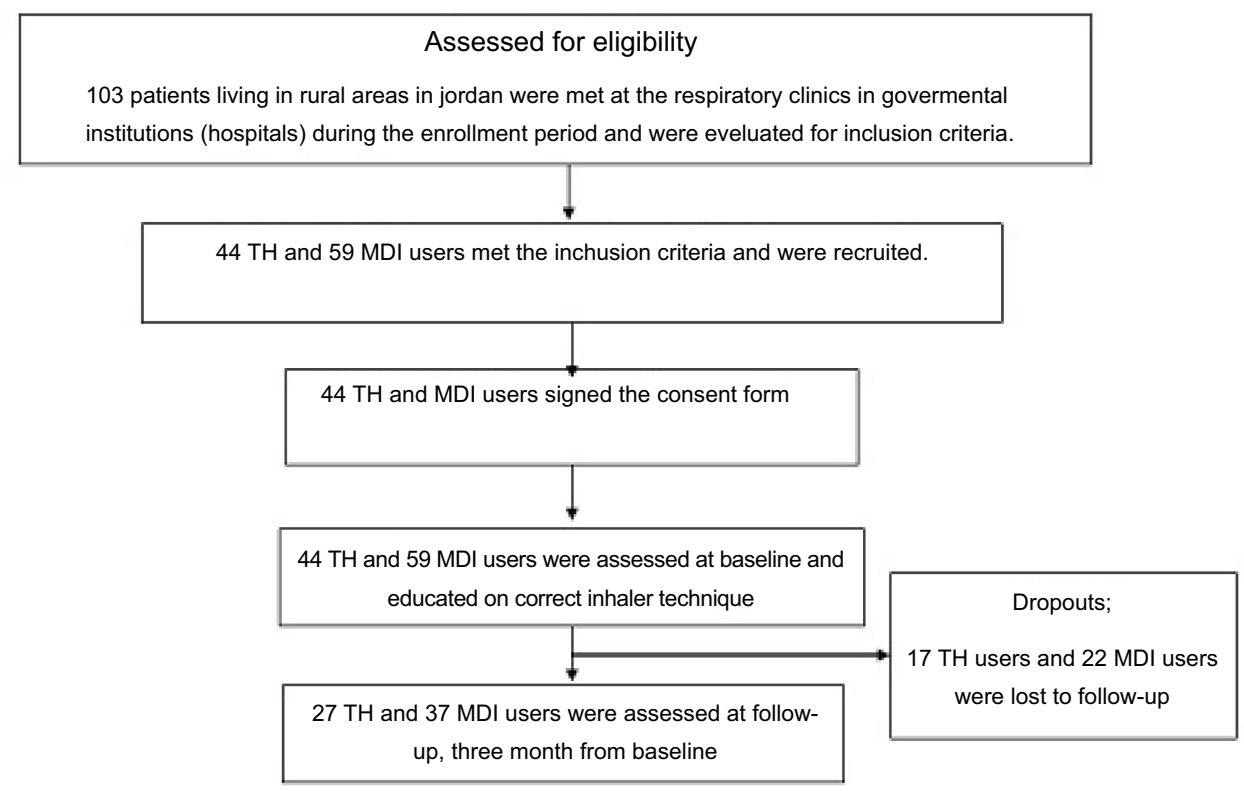

Figure I CONSORT diagram showing patient recruitment and retention in the study. Abbreviations: MDI, metered dose inhaler; $\mathrm{TH}$, turbuhaler. 
Table 2 Baseline demographic and asthma-related characteristics for TH $(n=44)$ and MDI $(n=59)$ users

\begin{tabular}{|c|c|c|c|c|}
\hline Variable & $\mathrm{TH}(\mathrm{n}=44)$ & $\operatorname{MDI}(n=59)$ & All $(n=103)$ & $p$-value \\
\hline Age (years), mean (SD) & $45.57(12.68)$ & $47.20(14.16)$ & $46.46(13.50)$ & 0.394 \\
\hline Gender (females), n (\%) & $34(77.3 \%)$ & $42(71.2 \%)$ & $76(73.8 \%)$ & 0.508 \\
\hline Number of family members, $n(\%)$ & & & & 0.512 \\
\hline $0-3$ & 7 (15.9\%) & $10(16.9 \%)$ & $17(16.5 \%)$ & \\
\hline $4-7$ & $14(31.8 \%)$ & $19(32.2 \%)$ & $33(32.0 \%)$ & \\
\hline $8-11$ & $14(31.8 \%)$ & $12(20.3 \%)$ & $26(25.2 \%)$ & \\
\hline $12-16$ & $9(20.5 \%)$ & $18(30.5 \%)$ & $27(26.2 \%)$ & \\
\hline Education level, n (\%) & & & & 0.706 \\
\hline Elementary school & $23(52.3 \%)$ & 37 (62.7\%) & $60(58.3 \%)$ & \\
\hline High school & $13(29.5 \%)$ & $15(25.4 \%)$ & $28(27.2 \%)$ & \\
\hline College & $4(9.1 \%)$ & $3(5.1 \%)$ & $7(6.8 \%)$ & \\
\hline University & $4(9.1 \%)$ & $4(6.8 \%)$ & $8(7.8 \%)$ & \\
\hline $\begin{array}{l}\text { Marital status, n (\%) } \\
\text { (married:widowed:divorced:single) }\end{array}$ & $\begin{array}{l}28: 6: 2: 8 \\
(63.6: 13.6: 4.5: 18.2)\end{array}$ & $52: 3: 2: 2(80.1: 5.1: 3.4: 3.4)$ & $\begin{array}{l}80: 9: 4: 10 \\
(77.7: 8.7: 3.9: 9.7)\end{array}$ & 0.020 \\
\hline Working status, n (\%) & & & & 0.363 \\
\hline Employed & $8(18.2 \%)$ & $13(22 \%)$ & 21 (20.4\%) & \\
\hline Student & $2(4.5 \%)$ & $2(3.4 \%)$ & $4(3.9 \%)$ & \\
\hline Unemployed & $30(68.2 \%)$ & $43(72.9 \%)$ & $73(70.9 \%)$ & \\
\hline Retired & $4(9.1 \%)$ & $\mathrm{I}(\mathrm{I} .7 \%)$ & $5(4.9 \%)$ & \\
\hline $\begin{array}{l}\text { Smoking status, } \mathrm{n}(\%) \\
\text { (nonsmoker:exsmoker:smoker) }\end{array}$ & $\begin{array}{l}30: 6: 8 \\
(68.2: 13.6: 18.2)\end{array}$ & $\begin{array}{l}38: 8: 13 \\
(64.4: 13.6: 22.0)\end{array}$ & $\begin{array}{l}68: 14: 21 \\
(66.0: 13.6: 20.4)\end{array}$ & 0.888 \\
\hline Duration of preventer use (years), mean (SD) & $11.70(9.7 \%)$ & $12.4 \mid(10.7 \%)$ & $12.1(10.3 \%)$ & 0.838 \\
\hline \multicolumn{5}{|l|}{ Asthma characteristics } \\
\hline $\begin{array}{l}\text { Onset of asthma, } n(\%) \\
\text { (infant ( } 0-2 \text { years):child ( } 3-18 \text { years):adult (>18 years)) }\end{array}$ & $\begin{array}{l}0: 11: 33 \\
(0.0: 25.0: 75.0)\end{array}$ & $\begin{array}{l}2: 7: 50 \\
(3.4: 11.9: 84.7)\end{array}$ & $\begin{array}{l}2: 18: 83 \\
(1.9: 17.5: 80.6)\end{array}$ & 0.118 \\
\hline Age of onset of asthma (years), mean (SD) & $31.50(15.72)$ & $32.68(14.06)$ & $32.5(15.1)$ & 0.105 \\
\hline Asthma control, $\mathrm{n}(\%)^{\mathrm{a}}$ & $1: 10: 33$ & $1: 10: 48$ & $2: 20: 81$ & 0.739 \\
\hline (well: moderate: poor) & $(2.3: 22.7: 75.0)$ & $(1.7: 16.9: 81.4)$ & $(1.9: 19.4: 78.6)$ & \\
\hline ACT score, mean $(S D)^{a}$ & $11.43(4.24)$ & $9.86(4.26)$ & $10.53(4.29)$ & 0.877 \\
\hline Reliever puffs/day, mean (SD) & $4.38(4.32)$ & $5.60(4.03)$ & $5.1(4.2)$ & 0.461 \\
\hline $\mathrm{FEV}, \%$ at baseline, mean (SD) & $54.57(19.47)$ & $54.20(17.87)$ & $54.36(18.47)$ & 0.922 \\
\hline
\end{tabular}

Notes: ${ }^{2}$ Asthma Control Test (ACT) scores out of 25 ; $\geq 20$, well-controlled asthma; 16-19, moderately controlled asthma; $\leq 15$, very poorly controlled asthma. ${ }^{44}$ Abbreviations: $\mathrm{FEV}_{1} \%$, forced expiratory volume in the first I second; MDI, metered dose inhaler; TH, turbuhaler.

Most patients reported that they like using their preventer inhalers $(77.67 \%)$, and the majority had no concerns with their inhalers $(86.40 \%)$. Few reported corticosteroid phobia (9.71\%); others did not like the taste (2.91\%) or thought their treatment was not effective $(0.97 \%)$.

Many were given over the counter medications by their pharmacist to help manage their asthma (21.36\%), and few used herbal treatment for the management of their condition $(4.85 \%)$.
Only $26.21 \%$ of patients performed a mouth wash following their preventer use, and few suffered from oral thrush $(6.80 \%)$.

When the patients were asked about breathing exercises used in asthma management, only $2.91 \%$ had heard of the lung exercises before. As for peak flow meter (PFM) use, only $3.88 \%$ had ever used one. Following explaining to the patients what an action plan was, none of the patients reported having had an action plan prepared by their doctor. 
Table 3 Comparing study outcomes (inhaler technique, FEV,\%, ACT, and reliever use) at baseline and end of study for study patients

\begin{tabular}{|c|c|c|c|}
\hline Variable & Baseline $(n=103)$ & End of study $(n=103)$ & $p$-value \\
\hline $\begin{array}{l}\text { Inhaler technique score, mean (SD) } \\
\text { TH }(n=44) \text {, score out of } 9 \\
\text { MDI }(n=59) \text {, score out of } 9 \\
\text { All }(n=103) \\
p \text {-value }\end{array}$ & $\begin{array}{l}5.48(\mathrm{SD}=1.97) \\
5.24(\mathrm{SD}=2.00) \\
5.34(\mathrm{SD}=1.98) \\
0.546\end{array}$ & $\begin{array}{l}7.39(\mathrm{SD}=1.19) \\
6.85(\mathrm{SD}=1.36) \\
7.08(\mathrm{SD}=1.31) \\
0.038\end{array}$ & $\begin{array}{l}<0.001 \\
<0.001 \\
<0.001\end{array}$ \\
\hline $\begin{array}{l}\text { Inhaler essential technique score, mean (SD) } \\
\text { TH }(n=44) \text {, score out of } 4 \\
\text { MDI }(n=59) \text {, score out of } 3\end{array}$ & $\begin{array}{l}2.37(S D=0.88) \\
1.84(S D=0.834)\end{array}$ & $\begin{array}{l}3.56(\mathrm{SD}=0.75) \\
2.19(\mathrm{SD}=0.62)\end{array}$ & $\begin{array}{l}<0.001 \\
0.002\end{array}$ \\
\hline $\begin{array}{l}\text { Correct inhaler technique, } n(\%) \\
\text { TH }(n=44) \\
\text { MDI }(n=59) \\
\text { All }(n=103) \\
p \text {-value }\end{array}$ & $\begin{array}{l}\text { I }(2.27 \%) \\
0(0.00 \%) \\
\text { I }(0.97 \%) \\
0.245\end{array}$ & $\begin{array}{l}14(31.82 \%) \\
15(25.42 \%) \\
29(28.16 \%) \\
0.475\end{array}$ & $\begin{array}{l}<0.001 \\
N A \\
<0.001\end{array}$ \\
\hline $\begin{array}{l}\text { Correct essential technique, } n(\%) \\
\text { TH }(n=44) \\
\text { MDI }(n=59) \\
\text { All }(n=103) \\
p \text {-value }\end{array}$ & $\begin{array}{l}3(6.82 \%) \\
4(6.78 \%) \\
7(6.80 \%) \\
0.994\end{array}$ & $\begin{array}{l}23(52.27 \%) \\
24(40.68 \%) \\
47(45.63 \%) \\
0.243\end{array}$ & $\begin{array}{l}<0.001 \\
<0.001 \\
<0.001\end{array}$ \\
\hline $\begin{array}{l}\text { FEV } \%^{a} \\
\text { TH }(n=44, n=27) \\
\text { MDI }(n=59, n=37) \\
\text { All }(n=103, n=64) \\
p \text {-value }\end{array}$ & $\begin{array}{l}54.57(\mathrm{SD}=19.47) \\
54.20(\mathrm{SD}=17.87) \\
54.36(\mathrm{SD}=18.47) \\
0.922\end{array}$ & $\begin{array}{l}59.52(\mathrm{SD}=20.20) \\
60.16(\mathrm{SD}=21.60) \\
59.89(\mathrm{SD}=20.86) \\
0.904\end{array}$ & $\begin{array}{l}0.097 \\
0.005 \\
0.001\end{array}$ \\
\hline $\begin{array}{l}\text { ACT }^{\mathrm{a}} \\
\text { TH }(n=44, n=27) \\
\text { MDI }(n=59, n=37) \\
\text { All }(n=103, n=64) \\
p \text {-value }\end{array}$ & $\begin{array}{l}11.5 \mid(S D=3.77) \\
10.22(S D=4.36) \\
10.77(S D=4.14) \\
0.067\end{array}$ & $\begin{array}{l}13.59(\mathrm{SD}=4.53) \\
13.24(\mathrm{SD}=4.25) \\
13.39(\mathrm{SD}=4.34) \\
0.753\end{array}$ & $\begin{array}{l}0.031 \\
<0.001 \\
<0.001\end{array}$ \\
\hline $\begin{array}{l}\text { Reliever use }^{\mathrm{a}} \\
\text { TH }(\mathrm{n}=44, \mathrm{n}=27) \\
\text { MDI }(\mathrm{n}=59, \mathrm{n}=37) \\
\text { All }(\mathrm{n}=103, \mathrm{n}=64) \\
\text {-value }\end{array}$ & $\begin{array}{l}6.36(\mathrm{SD}=4.46) \\
7.82(\mathrm{SD}=3.42) \\
7.21(\mathrm{SD}=3.93) \\
0.144\end{array}$ & $\begin{array}{l}4.21 \quad(S D=4.08) \\
5.14 \quad(S D=3.7 I) \\
4.744 \mid \quad(S D=3.87) \\
0.345\end{array}$ & $\begin{array}{l}0.009 \\
<0.001 \\
<0.001\end{array}$ \\
\hline
\end{tabular}

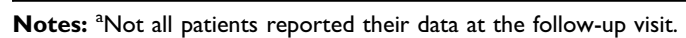

Abbreviations: ACT, Asthma Control Test; FEV $\%$, forced expiratory volume in the first I second; MDI, metered dose inhaler; NA, not applicable; TH, turbuhaler.

\section{Follow-up assessment}

Improvement in asthma severity was significant for $\mathrm{TH}$ and MDI users (Figure 2). Both $\mathrm{TH}$ and MDI users showed similar improvements in ACT score across the study, with no reported differences between the inhalers at follow-up (Table 3). The majority of TH users were categorized in the poorly controlled asthma category (15/ 27), while the rest had either moderately controlled asthma $(8 / 27)$ or well-controlled asthma (4/27). The majority of MDI users had poorly controlled asthma (24/37), the rest had either moderately controlled (10/37) or well-controlled (3/37) asthma.

The minimum clinically important difference in ACT scores is 3 points. Patients using the MDI achieved 3.03 ( \pm 4.30$)$, which is a clinically significant improvement in control. Patients using the TH achieved a $2.07( \pm 4.72)$ improvement, which although statistically significant $(p=0.031)$ was not clinically significant. ${ }^{33}$

The $\mathrm{FEV}_{1} \%$ improved significantly for MDI users $(p=0.005)$ but not for TH users $(p=0.097)$. 
Table 4 Past inhaler technique education reported by study participants at baseline $(n=103)$

\begin{tabular}{|c|c|c|c|c|}
\hline & TH $(n=44)$ & MDI (n=59) & All $(n=103)$ & $\begin{array}{l}p- \\
\text { value }\end{array}$ \\
\hline Why did you choose to use this type of inhaler?, n (\%) & & & & 0.927 \\
\hline Specialist advice & $42(95.45 \%)$ & $56(94.92 \%)$ & $98(95.15 \%)$ & \\
\hline Pharmacist advice & I (2.27\%) & $\mathrm{I}(1.69 \%)$ & $2(4.85 \%)$ & \\
\hline Family advice & I (2.27\%) & $2(3.39 \%)$ & $3(2.91 \%)$ & \\
\hline $\begin{array}{l}\text { Have you ever been provided with education or advice about } \\
\text { how to use your inhaler? "Yes", n (\%) }\end{array}$ & $44(100 \%)$ & $59(100 \%)$ & $103(100 \%)$ & NA \\
\hline If yes, was this by: & & & & 0.295 \\
\hline Regular doctor & $0(0.00 \%)$ & $3(50.85 \%)$ & $3(2.91 \%)$ & \\
\hline Pharmacist & I (2.27\%) & $0(0.00 \%)$ & I (0.97\%) & \\
\hline Medical center & $0(0.00 \%)$ & I (I.69\%) & I (0.97\%) & \\
\hline Specialist & $5(11.36 \%)$ & $3(5.08 \%)$ & $8(7.77 \%)$ & \\
\hline Hospital clinic & 37 (84.09\%) & 49 (83.08\%) & $86(83.50 \%)$ & \\
\hline Other people & I (2.27\%) & $3(2.08 \%)$ & $4(3.88 \%)$ & \\
\hline If yes, what was the type of counseling?, $n(\%)$ & & & & 0.147 \\
\hline Verbal information & $22(50.00 \%)$ & $33(55.93 \%)$ & $55(53.40 \%)$ & \\
\hline Written information & $0(0.00 \%)$ & I (I.69\%) & I (0.97\%) & \\
\hline Physical demonstration & $19(43.18 \%)$ & $15(25.42 \%)$ & $34(33.00 \%)$ & \\
\hline Cannot recall & $3(6.82 \%)$ & $10(16.95 \%)$ & $13(12.62 \%)$ & \\
\hline If yes, when did you receive this information or advice?, n (\%) & & & & 0.263 \\
\hline When you first got your inhaler & $36(81.82 \%)$ & $37(62.71 \%)$ & $73(70.87 \%)$ & \\
\hline After you started using your inhaler & I (2.27\%) & $2(3.40 \%)$ & $3(2.91 \%)$ & \\
\hline At some other time & $4(9.09 \%)$ & $6(10.17 \%)$ & $10(9.71 \%)$ & \\
\hline After requesting information on how to use your inhaler & $0(0.00 \%)$ & $\mathrm{I}(69 \%)$ & I (0.97\%) & \\
\hline Cannot recall & $3(6.82 \%)$ & $13(22.03 \%)$ & $16(15.53 \%)$ & \\
\hline $\begin{array}{l}\text { Have you received any information or advice about how to use } \\
\text { your inhaler in the last } 12 \text { months? "Yes", n (\%) }\end{array}$ & $3(6.82 \%)$ & $10(16.95 \%)$ & $13(12.62 \%)$ & 0.126 \\
\hline Has anyone ever checked the way you use your inhaler? “Yes”, n (\%) & $3(6.82 \%)$ & $4(6.78 \%)$ & $7(6.80 \%)$ & 0.994 \\
\hline $\begin{array}{l}\text { Do you think that you use your inhaler correctly?, n (\%) } \\
\text { (no:yes:don't know) }\end{array}$ & $\begin{array}{l}8: 21: 11 \\
(18.18: 47.73: 25.00)\end{array}$ & $\begin{array}{l}3: 22: 43 \\
(5.08: 37.29: 72.88)\end{array}$ & $\begin{array}{l}\text { II:34:49 } \\
(10.68: 33.01: 47.57)\end{array}$ & 0.022 \\
\hline $\begin{array}{l}\text { Do you like using your inhaler for your asthma management? } \\
\text { "Yes", n (\%) }\end{array}$ & $32(72.73 \%)$ & $48(81.36 \%)$ & $80(77.67 \%)$ & 0.298 \\
\hline Do you have concerns with using your inhaler?, n (\%) & & & & 0.363 \\
\hline No & $40(90.90 \%)$ & 49 (83.05\%) & $89(86.40 \%)$ & \\
\hline Corticosteroid phobia & $4(9.09 \%)$ & $6(10.17 \%)$ & $10(9.71 \%)$ & \\
\hline Bad taste & $0(0.00 \%)$ & $3(5.08 \%)$ & $3(2.91 \%)$ & \\
\hline Not effective & $0(0.00 \%)$ & I (I.69\%) & I (0.97\%) & \\
\hline $\begin{array}{l}\text { Were you given over the counter medications by your phar- } \\
\text { macist to help manage your asthma? "Yes", n (\%) }\end{array}$ & $9(20.45 \%)$ & $13(22.03 \%)$ & $22(21.36 \%)$ & 0.847 \\
\hline $\begin{array}{l}\text { Do you use any herbal treatment for the management of your } \\
\text { asthma? "Yes", n (\%) }\end{array}$ & $2(4.55 \%)$ & $3(5.08 \%)$ & $5(4.85 \%)$ & 0.900 \\
\hline Do you perform mouth rinsing after using your inhaler? “Yes”, n (\%) & $12(27.27 \%)$ & $15(25.42 \%)$ & $27(26.21 \%)$ & 0.833 \\
\hline Do you suffer from oral thrush? "Yes", n (\%) & I (2.27\%) & $6(10.17 \%)$ & 7 (6.80\%) & 0.152 \\
\hline
\end{tabular}

(Continued) 
Table 4 (Continued).

\begin{tabular}{|l|l|l|l|l|}
\hline & TH (n=44) & MDI (n=59) & All (n=103) & $\begin{array}{l}\text { p- } \\
\text { value }\end{array}$ \\
\hline $\begin{array}{l}\text { Have you heard of the breathing exercise to manage your } \\
\text { asthma? "Yes", n (\%) }\end{array}$ & I (2.27\%) & $2(3.39 \%)$ & $3(2.91 \%)$ & 0.739 \\
\hline $\begin{array}{l}\text { Do you have an asthma action plan } \\
\text { "Yes", n (\%) }\end{array}$ & $0(0.00 \%)$ & $0(0.00 \%)$ & $0(0.00 \%)$ \\
\hline Have you ever used a PFM before? "Yes", n (\%) & $3(6.82 \%)$ & $1(1.69 \%)$ & $4(3.88 \%)$ & NA \\
\hline
\end{tabular}

Abbreviations: ACT, Asthma Control Test; FEV,\%, forced expiratory volume in the first I second; MDI, metered dose inhaler; NA, not applicable; PFM, peak flow meter; $\mathrm{TH}$, turbuhaler.

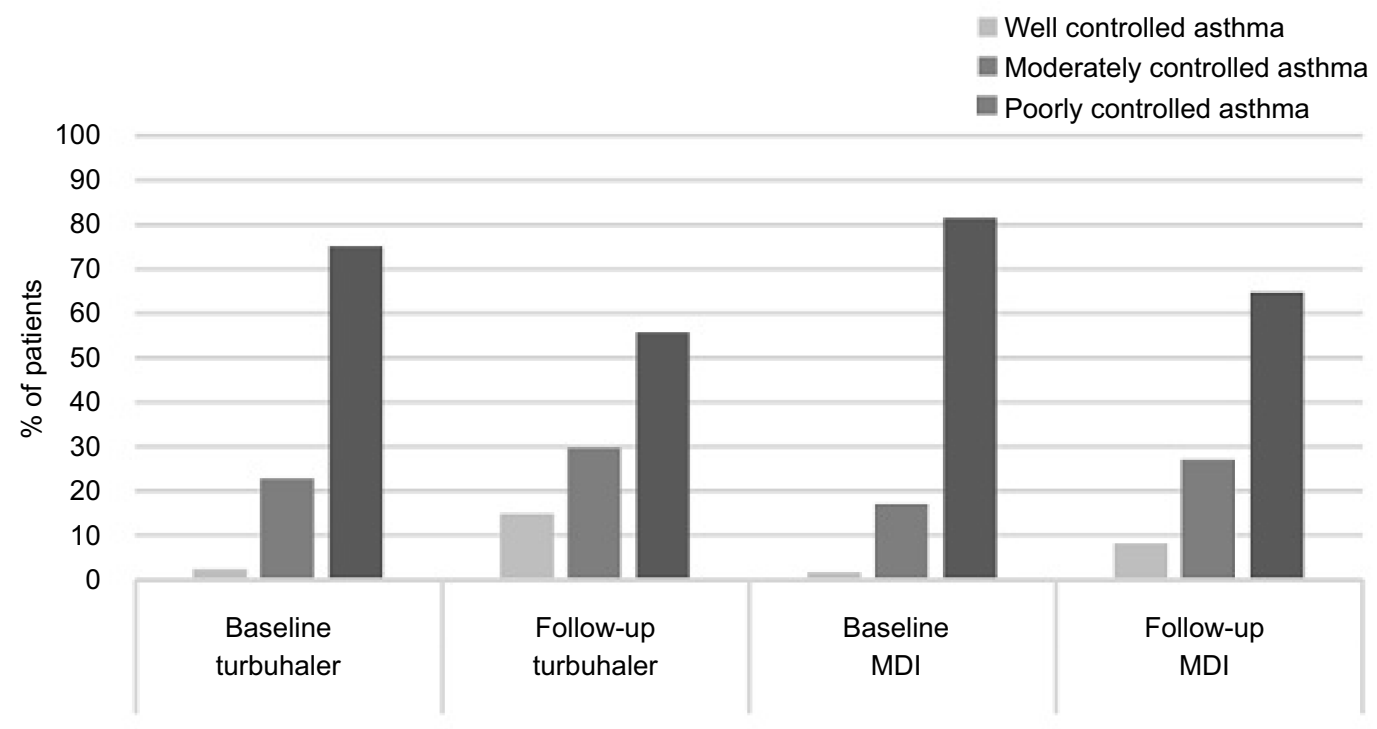

Figure 2 Proportion of patients categorized according to asthma severity at baseline (TH, n=44; MDI, n=59) and follow-up (TH, $n=27 ; M D I, n=37)$.

Abbreviations: $\mathrm{MDI}$, metered dose inhaler; $\mathrm{TH}$, turbuhaler.

Inhaler reliever puffs decreased significantly for $\mathrm{TH}$ and MDI users (Table 3). A mean difference of -2.16 (4.00) for TH users and -2.69 (3.35) for MDI users was observed, with no significant difference between the inhaler groups $(p=0.568)$.

All patients were seen at follow-up, which was within a similar time period for TH $(3.72 \pm 0.994$ months $)$ and MDI ( $3.59 \pm 0.70$ months) users $(p=0.547)$. Assessment showed a significant improvement in inhaler technique scores from baseline, correct technique, and correct essential technique (Table 3). No significant differences between TH and MDI users were reported at follow-up.

\section{Correlation results}

Significant correlations were identified between improvements in ACT scores and improvements in $\mathrm{FEV}_{1} \%$ scores $(\mathrm{r}=0.612, p<0.001)$, and improvement in $\mathrm{FEV}_{1} \%$ scores and decrease in reliever use, puffs/day $(\mathrm{r}=-0.261$, $p=0.037$ ). No significant correlation between ACT score improvements and improvements in inhaler technique scores was found $(\mathrm{r}=0.122, p=0.338)$, nor between improvements in $\mathrm{FEV}_{1} \%$ scores and improvement in inhaler technique scores $(\mathrm{r}=0.103, p=0.418)$ or between decrease in reliever use, puffs/day, and inhaler technique scores $(\mathrm{r}=-0.177, p=0.163)$.

\section{Multiple regression for primary variable}

Results from the multivariable regression analysis, with change in ACT scores across the study being the dependent variable (mean score of ACT after 3 months minus mean score of ACT at baseline), showed that ACT scores 
Table 5 Summary of the regression model obtained for the dependent variable, ACT score change across the study $(n=64)$

\begin{tabular}{|c|c|c|c|}
\hline Variable & $\boldsymbol{\beta}$ & $\mathbf{t}$ & $p$-value \\
\hline Inhaler type (TH or MDI) & -0.039 & -0.476 & 0.636 \\
\hline Age & -0.036 & -0.402 & 0.689 \\
\hline Gender & -0.071 & -0.851 & 0.398 \\
\hline Change in inhaler technique mean score & 0.050 & 0.596 & 0.554 \\
\hline ACT scores at baseline & -0.507 & -6.039 & $<0.001$ \\
\hline Change in $\mathrm{FEV}_{1} \%$ across the study & 0.604 & 7.375 & $<0.001$ \\
\hline
\end{tabular}

Notes: This table shows the output from a multivariable regression analysis of Asthma Control Test (ACT) score improvement across the study (overall fit of the model was $\left.R^{2}=0.631, p<0.001\right)$.

Abbreviations: $\beta$, standardized regression coefficient; $F E V_{1} \%$, forced expiratory volume in the first I second; MDI, metered dose inhaler; $\mathrm{TH}$, turbuhaler.

at baseline and change in $\mathrm{FEV}_{1} \%$ scores across the study were the only variables that showed significant association with the dependent variable $\left(\mathrm{R}^{2}=0.631, p<0.001\right.$; Table 5).

\section{Individual steps technique}

For patients using the MDI, the majority of patients did not perform the fourth step in the technique checklist (keeping the head upright or slightly tilted). Continuing with slow and deep inhalation and holding breath for 5 seconds were also incorrectly performed by the majority of patients (Figure 3 ). At follow-up, significant improvements were found for patients performing step 1 "removing the mouthpiece cover and shaking" ( $p=0.045)$, step 2 "holding the inhaler upright" $(p<0.001)$, step 4 "keeping head upright or slightly tilted" $(p<0.001)$, and step 6 "inhaling slowly and pressing canister" $(p<0.001)$. Steps 1 and 6 are essential steps. No significant improvement in step 7 "continue slow and deep inhalation", an essential step, was found $(p=0.472)$.

TH users demonstrated weakness in performing step 2 "keeping inhaler upright", step 4 "exhaling to residual volume", and step 9 "exhaling away from the mouthpiece" (Figure 4). These steps were significantly improved at followup ( $p<0.001$ for the 3 steps). Other steps for which patients demonstrated significant improvement at follow-up included step 3 "rotating the grip until a click is heard" $(p=0.005)$, step 5 "exhaling away from the mouthpiece" $(p<0.001)$, and step 7 "inhale forcefully and deeply" ( $p=0.001)$. It is important to note that steps 2,3 , and 7 are essential steps.

\section{Discussion}

This pre-post interventional study provides an important insight into the asthma management of patients with asthma living in the rural areas in Jordan. Results indicate that most patients living in the rural areas have poor inhaler technique, poorly controlled asthma (low ACT scores), low $\mathrm{FEV}_{1} \%$ scores, and high use of reliever treatment. Important aspects of asthma management, such as having an action plan and using a PFM, are missing. To the best of our knowledge, this is the first study to examine asthma management in rural Jordan. This study engaged the pharmacist in a simple educational intervention on inhaler technique in the rural areas in Jordan. The study revealed the effectiveness of a pharmacist-led simple educational intervention on inhaler technique and clinical outcomes for patients with asthma, including asthma control, $\mathrm{FEV}_{1} \%$, and reliever use.

Jordan's inequality levels with regards to health-care services are low compared to international standards; ${ }^{22}$ Jordan achieved nearly universal coverage and good availability of health services, without differences across place of residence or income quintile (any of 5 equal groups into which a population can be divided according to the distribution of values of a particular variable). ${ }^{22}$ However, while there are few differences in terms of access to health and education, there may be some quality of services issues that have not been evaluated, due to the limited information included in the data collected. ${ }^{22}$ Usually, smaller rural health-care centers do not have the resources or facilities to assess and educate patients on the management of their condition. ${ }^{35}$ The rural environment presents an issue with regards to distance to the hospital during an emergency. ${ }^{36}$ Patients recruited in this study lived with unmanaged asthma, with a $\mathrm{FEV}_{1} \%$ of $54.36 \pm 18.47$. A low $\mathrm{FEV}_{1} \%$ $<60 \%$ predicted is a potentially modifiable independent risk factor for exacerbations besides being a risk factor for developing fixed airflow limitation. ${ }^{37}$ Patients had a very low ACT score $(10.77 \pm 4.14)$ and high daily intake of reliever treatment $(7.21 \pm 3.93$ puffs/day). A lot can be and should be done for this population. Although in such communities pharmacists were ranked, along with the doctors, as the most consulted health-care professionals, ${ }^{36}$ this study revealed a minimal role of the pharmacist in managing patients with asthma in rural Jordan. It would be expected for pharmacists to play a substantial role in maintaining the health of patients in the rural areas because of limited access to the primary health-care facilities. ${ }^{38}$ In addition, patients in rural areas tend to visit the pharmacist before going to the specialist or hospital emergency department in an attempt to save on consultation fees. Studies from around the world have shown that community pharmacies present a suitable facility for assessing and educating patients on inhaler technique. ${ }^{27,39}$ A recent literature review study 


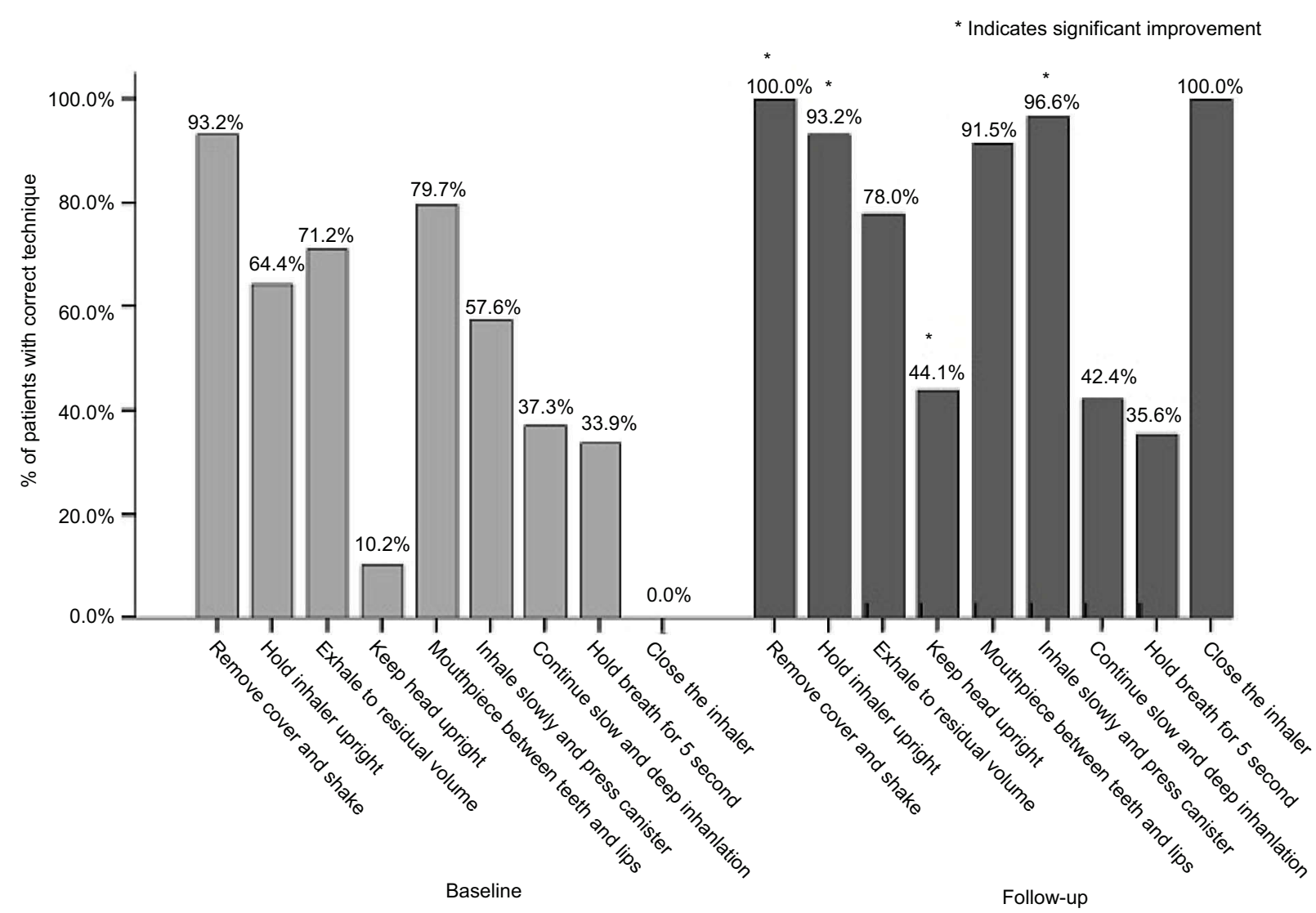

Figure 3 Proportion of metered dose inhaler users who demonstrated each step in the inhaler technique checklist correctly at baseline and follow-up ( $=59$ ).

reported that pharmacists can play an effective role in screening for poorly controlled asthma and in delivering management interventions. ${ }^{40}$ In this study, patients reported that specialists and hospital clinics contributed the most to teaching patients on correct inhaler use. This could be the reason behind patients receiving education on inhaler technique only when they first started using their inhalers, and less than $13 \%$ of them received information or advice about how to use their inhalers in the last 12 months prior to this study. No inhaler technique review during the previous year has been associated with patients making serious errors; factors significantly associated with serious errors included asthma-related hospitalization in the previous year and poor asthma control in the previous 4 weeks. ${ }^{41}$ In view of the observed improved inhaler technique seen 3 months after correcting the patient's inhaler technique at baseline, the development of this pharmacist-provided education led to long-term improvement in patients' inhaler technique.

When it comes to achieving and sustaining the patient's correct inhaler technique, this study has demonstrated that while assessing and educating is important in achieving correct inhaler technique, reassessing and re-educating are important in maintaining the correct technique achieved. ${ }^{28}$ In this study, patients were assessed and then educated to have correct inhaler technique at baseline. All patients demonstrated correct technique following education at baseline. The 3-month follow-up period was associated with worsening of inhaler technique, an outcome that is in concordance with previous findings. ${ }^{42}$ Hence, reassessing and re-educating patients on correct inhaler technique needs to be a regular and ongoing process; pharmacists are well placed to do this, because unlike specialists they can see the patient every time an inhaler is dispensed.

It is noteworthy that this study was conducted in the respiratory clinics of three governmental institutions (hospitals) accessible by the rural population. Supply of medications at these hospitals complied with the policies and procedures governing the provision of essential asthma medications. Hence, asthmatic patients who were recruited to the study had their inhalers available on time via the hospital's pharmacy.

Inhaler technique checklists developed from previous published work $^{18,34}$ were used in the assessment of patients' inhaler technique. Many studies used checklists 


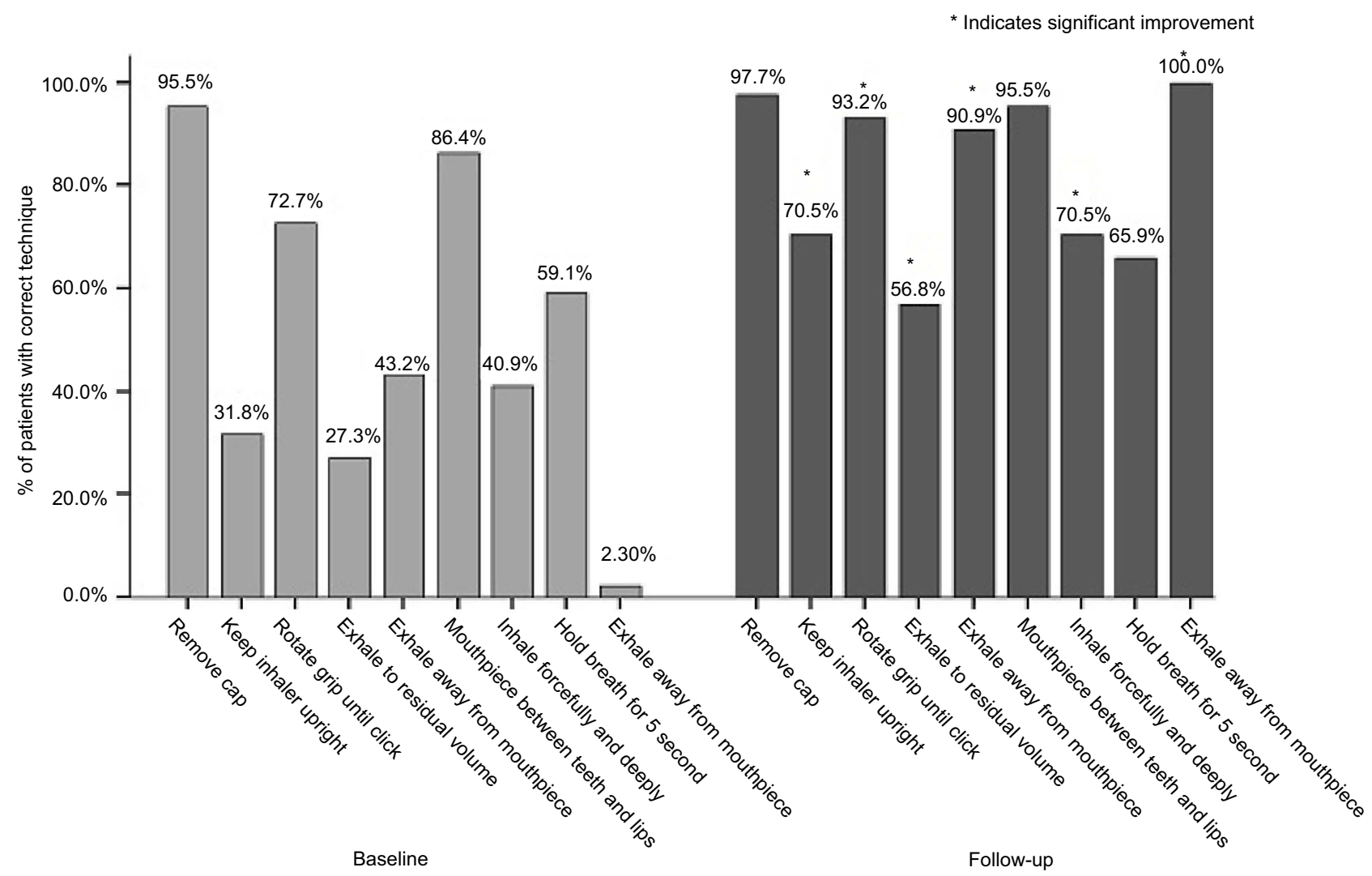

Figure 4 Proportion of turbuhaler users who demonstrated each step in the inhaler technique checklist correctly at baseline and follow-up ( $\mathrm{n}=44$ ).

to assess patients' inhaler technique. ${ }^{34,43}$ This study is unique in that the researcher used the checklist to highlight problematic steps performed by the patient during assessment, explaining the importance of performing that step correctly. This way, the educational session was individualized based on each patient's need.

In order to explore whether the improvement in inhaler technique detected in this study was the main driver in the clinical improvements, shown via the ACT score improvement, $\mathrm{FEV}_{1} \%$ score improvement, and decreased reliever use, further exploratory analysis was carried out. Results from both correlation and multiple regression statistics showed no significant association between improvement in the inhaler technique and clinical outcomes of patients. Previous reports stated an association between reducing inhaler technique errors over time and improved asthma outcomes. ${ }^{41,43}$ This contrasts with the present study, which showed no significant association between better inhaler technique scores and higher ACT scores. A previous study also found no relationship between inhaler technique improvement and improvements seen in clinical outcomes. ${ }^{5}$ The fact that the majority of participants in this study, whether they were using the MDI or the TH, were categorized in the "very poor asthma control" category could have affected the results. ${ }^{5}$ In addition, all patients were educated in correct inhaler technique at baseline, hence, other variables could have played a role in the ACT improvements seen at follow-up.

Asthma control was assessed using the ACT score, which is recommended in guidelines, ${ }^{1}$ and has shown a strong correlation with physician-assessed asthma control, ${ }^{30,44}$ $\mathrm{FEV}_{1} \%$, and asthma-specific health-related quality of life. In addition, ACT was shown to properly predict Global Initiative for Asthma-defined uncontrolled asthma in $81 \%$ of adult patients. ${ }^{31}$ We recommend the routine use of the ACT in Jordan for clinical assessment of asthma control, especially since spirometry services are not readily available in all health-care settings. ${ }^{45}$ Interestingly, significant correlations between $\mathrm{ACT}$ scores, $\mathrm{FEV}_{1} \%$, and decreased reliever use were revealed in this study.

It is well known that asthmatic patients need to stay away from allergens and irritants of the respiratory tract, and can benefit from performing regular exercise to improve the function of their lungs, in addition to having a PFM and an action plan to better manage their condition. ${ }^{46}$ Many of the patients in 
this study were smokers, with no knowledge of the lung exercises and the importance of having a PFM and an action plan. With $79 \%$ of these patients suffering from poorly controlled asthma, using a PFM and having an action plan is needed according to the latest Global Initiative for Asthma guidelines. ${ }^{1}$ Improvements in these areas in asthma education and use of written action plans are required in the rural setting. ${ }^{36}$

The results of this study revealed an interesting and important facet with regards to asthma patients' inhaler technique. Asthma patients are "unaware" of their incorrect use of inhaler devices. Many of the patients in this study $(67 \%)$ believed that they were using their inhalers correctly at baseline, whereas results showed that only $1 \%$ were actually using their inhalers correctly. This finding is not new, as previous studies have drawn attention to this important problem. ${ }^{47-49}$ When patients believe that they use their inhalers correctly, they may not approach their pharmacists or any other health-care professional for a review of their inhaler technique. Hence, pharmacists need to be informed of the "unawareness" of the asthma patient and his/her inhaler technique, and of the necessity to review and educate every patient purchasing an inhaler device.

This accomplished work confirmed that assessment and education is important in attaining correct inhaler technique, and reassessment and re-education is important to preserve correct technique. All patients had correct technique before they left the clinics at baseline. A 3-month interval led to $46 \%$ of the patients demonstrating correct essential technique and $28 \%$ demonstrating correct technique. This is the reason why a periodical repeated assessment as a follow-up is recommended.

Certain steps in the TH and MDI checklists were found to be more prone to error than others when the use of the devices was demonstrated by patients. MDI users incorrectly demonstrated step 4 "keeping the head upright or slightly tilted", step 7 "continuing with slow and deep inhalation", and step 8 "holding breath for 5 seconds". For the TH, besides not holding the device upright, the key maneuvers performed incorrectly by the majority of users included "exhaling to residual volume", "exhaling away from the inhaler", and "holding breath for 5 seconds". These results are in agreement with findings of many previous studies. ${ }^{19,41}$ Deserving of note, a recent study incorporated a device titled the "INhaler Compliance Assessment-adapted inhaler" to collect audio files from patients' use of their inhalers, providing quantitative information on the time and technique of inhaler use; results indicated that only $20 \%$ of patients used their inhaler in the correct manner at the correct interval. Out of all errors, errors in drug priming accounted for $11 \%$, exhalation into the inhaler mouthpiece after dose actuation and before inhalation acounted for $18 \%$, while errors in inhalation accounted for $46 \%$ with low peak inspiratory flow rate being the most common inhaler technique error. ${ }^{39}$ The implication of knowing which steps in the MDI and TH checklists are most prone to be performed incorrectly by the patients is vital, as healthcare providers can be advised to provide further attention to these steps when educating their patients on correct inhaler technique. Loading of the TH is a harder maneuver to achieve correctly than loading of the MDI. ${ }^{18}$ This may not be related to the difficulty associated with this step, but rather to the fact that there are specific instructions associated with the loading of the TH (ie, loading the inhaler while keeping it upright). This is a key issue in the $\mathrm{TH}$ technique because the $\mathrm{TH}$ is an open reservoir inhaler, so, if loaded while not held upright, a suboptimal amount of the dose would be loaded for inhalation.

When it comes to the generalizability of this study, there are several issues to be considered. The study population consisted of patients with asthma, 79\% of whom were in the poorly controlled asthma category. Although this is common among asthma clinical studies, ${ }^{49}$ in terms of the outcomes it is possible that, had the sample been closer to representing the general population in terms of asthma severity, then the improvements seen in the clinical outcomes could have been different. Nevertheless, based on the findings of this study and previous studies, incorrect inhaler technique is a critical issue, and regardless of the impact on clinical outcomes, educating asthma patients on the correct use of inhaler devices is essential.

\section{Strengths and limitations}

Strengths of the study include checking inhaler technique using published checklists which afford reproducible scores. Inhaler technique checklists were used not only to assess patients' inhaler technique, but also as tools to educate patients on the importance of each step in the technique of using their inhalers. In the checklists used to assess patients' inhaler technique in this study, certain steps were identified to be "essential". Therefore, the number of patients with correct technique and correct essential technique was inspected in this study. Results have shown that the number of patients with incorrect $\mathrm{TH}$ and MDI technique was not significantly different at baseline and at follow-up.

The usual limitation of this type of study design included the Hawthorne effect, as patients know that their inhaler technique is being assessed and so they tend 
to change their behavior that might have altered the technique they usually follow at home. ${ }^{4}$

\section{Conclusion}

Asthmatic patients living in rural areas in Jordan have poor inhaler technique, ACT scores, $\mathrm{FEV}_{1} \%$ scores, and high use of reliever medications. Pharmacists have a minimal role in helping patients manage their condition. This study was conducted in the respiratory clinics by clinical pharmacists, and adds to the body of literature investigating a variety of different asthma interventions delivered by pharmacists in the rural areas around the world. The intervention delivered led to improved inhaler technique and lung function and reduced reliever use. The implications of this study from a feasibility perspective are that, with minimal training, pharmacists in the rural areas would be able to implement a device training educational session to all patients with asthma, using DPIs and MDIs, with the outcome of improvements in asthma management.

\section{Data availability}

The datasets generated during and/or analyzed during the current study are available from the corresponding author on reasonable request.

\section{Acknowledgments}

The authors are grateful to the Applied Science Private University Amman, Jordan, for the full financial support granted to this research project. The authors wish to thank the health-care professionals and patients who participated in this study.

\section{Disclosure}

Placebo inhalers were provided by AstraZeneca plc (London, UK) and GlaxoSmithKline plc (London, UK). The authors report no other conflicts of interest in this work.

\section{References}

1. Global Initiative for Asthma (GINA) Global strategy for asthma management and prevention report 2018. Available from: https:// ginasthma.org/wp-content/uploads/2018/04/wms-GINA-2018-reportV1.3-002.pdf. Accessed April 2019.

2. Abuekteish F, Alwash R, Hassan M, Daoud AS. Prevalence of asthma and wheeze in primary school children in northern Jordan. Ann Trop Paediatr. 1996;16(3):227-231.

3. Abu-Ekteish F, Otoom S, Shehabi I. Prevalence of asthma in Jordan: comparison between Bedouins and urban schoolchildren using the International Study of Asthma and Allergies in Childhood phase III protocol. Allergy Asthma Proc. 2009;30(2):181-185. doi:10.2500/ aap.2009.30.3208
4. Basheti IA, Obeidat NM, Reddel HK. Effect of novel inhaler technique reminder labels on the retention of inhaler technique skills in asthma: a single-blind randomized controlled trial. NPJ Prim Care Respir Med. 2017;27(1):9. doi:10.1038/s41533-017-0011-4

5. Basheti IA, Obeidat NM, Ammari WG, Reddel HK. Associations between inhaler technique and asthma control among asthma patients using pressurised MDIs and DPIs. Int J Tuberc Lung Dis. 2016;20 (5):689-695. doi:10.5588/ijtld.15.0557

6. Janezic A, Locatelli I, Kos M. Inhalation technique and asthma outcomes with different corticosteroid-containing inhaler devices. $J$ Asthma. 2019;27:1-9. doi:10.1080/02770903.2019.1591442

7. Price D, Bosnic-Anticevich S, Briggs A, et al. Inhaler competence in asthma: common errors, barriers to use and recommended solutions. Respir Med. 2013;107(1):37-46. doi:10.1016/j.rmed.2012.09.017

8. Basheti IA, Qunaibi EA, Hamadi SA, Reddel HK. Inhaler technique training and health-care professionals: effective long-term solution for a current problem. Respir Care. 2014;59(11):1716-1725. doi: $10.4187 /$ respcare. 02671

9. Basheti IA, Armour CL, Reddel HK, Bosnic-Anticevich SZ. Longterm maintenance of pharmacists' inhaler technique demonstration skills. Am J Pharm Educ. 2009;73(2):32. doi:10.5688/aj730232

10. Walley T, Bundred P, Rannard A, Bogg J. Challenges in changing to non-chlorofluorocarbon inhalers in the treatment of asthma. Postgrad Med J. 1999;75(890):710-714. doi:10.1136/pgmj.75.890.710

11. Roche N, Huchon GJ. Rationale for the choice of an aerosol delivery system. J Aerosol Med. 2000;13(4):393-404. doi:10.1089/ jam.2000.13.393

12. Borgstrom L, Asking L, Thorsson L. Idealhalers or realhalers? A comparison of Diskus and Turbuhaler. Int J Clin Pract. 2005;59 (12):1488-1495. doi:10.1111/j.1368-5031.2005.00747.x

13. Borgstrom L. On the use of dry powder inhalers in situations perceived as constrained. $J$ Aerosol Med. 2001;14(3):281-287. doi:10.1089/089426801316970231

14. van der Palen J. Peak inspiratory flow through diskus and turbuhaler, measured by means of a peak inspiratory flow meter (In-Check DIAL). Respir Med. 2003;97(3):285-289.

15. Chrystyn $\mathrm{H}$. Effects of device design on patient compliance: comparing the same drug in different devices. In: Dalby RN, Byron PR, Peart J, Suman JD, editors. Respiratory Drug Delivery Europe. Richmond, VA, USA:Davis Healthcare International Publishing, Virginia Commonwealth University; 2009:105-116.

16. Azouz W, Chrystyn H. Clarifying the dilemmas about inhalation techniques for dry powder inhalers: integrating science with clinical practice. Prim Care Respir J. 2012;21(2):208-213. doi:10.4104/pcrj.2012.00010

17. Bosnic-Anticevich SZ, Sinha H, So S, Reddel HK. Metered-dose inhaler technique: the effect of two educational interventions delivered in community pharmacy over time. J Asthma. 2010;47(3):251256. doi:10.3109/02770900903580843

18. van der Palen J, Klein JJ, Schildkamp AM. Comparison of a new multidose powder inhaler (Diskus/Accuhaler) and the Turbuhaler regarding preference and ease of use. J Asthma. 1998;35(2):147-152.

19. Basheti IA, Qunaibi E, Bosnic-Anticevich SZ, et al. User error with Diskus and Turbuhaler by asthma patients and pharmacists in Jordan and Australia. Respir Care. 2011;56(12):1916-1923. doi:10.4187/ respcare. 01205

20. Halwani R, Vazquez-Tello A, Horanieh N, et al. Risk factors hindering asthma symptom control in Saudi children and adolescents. Pediatr Int. 2017;59(6):661-668. doi:10.1111/ped.13268

21. Urbstonaitis R, Deshpande M, Arnoldi J. Asthma and health related quality of life in late midlife adults. Res Social Adm Pharm. 2019;15 (1):61-69. doi:10.1016/j.sapharm.2018.1003.1003

22. United Nations Development Programme (UNDP) and United Nations Children's Fund (UNICEF). Socio-economicInequality in Jordan. 2015. Available from: https://www.undp.org/content/dam/jor dan/docs/Poverty/UNDP\%20Socio\%20economic\%20Inequality\% 20in\%20Jordan\%20English.pdf. Accessed April 2019. 
23. Valet RS, Perry TT, Hartert TV. Rural health disparities in asthma care and outcomes. J Allergy Clin Immunol. 2009;123(6):1220-1225. doi:10.1016/j.jaci.2008.1212.1131

24. Cicutto L, Dingae MB, Langmack EL. Improving asthma care in rural primary care practices: a performance improvement project. $J$ Contin Educ Health Prof. 2014;34(4):205-214. doi:10.1002/chp.21254

25. Crompton GK, Barnes PJ, Broeders M, et al. The need to improve inhalation technique in Europe: a report from the Aerosol Drug Management Improvement Team. Respir Med. 2006;100(9):14791494. doi:10.1016/j.rmed.2006.01.008

26. Basheti IA, Reddel HK, Armour CL, Bosnic-Anticevich SZ. Counseling about turbuhaler technique: needs assessment and effective strategies for community pharmacists. Respir Care. 2005;50(5):617-623.

27. Basheti IA, Reddel HK, Armour CL, Bosnic-Anticevich SZ. Improved asthma outcomes with a simple inhaler technique intervention by community pharmacists. J Allergy Clin Immunol. 2007;119 (6):1537-1538. doi:10.1016/j.jaci.2007.02.037

28. Basheti IA, Armour CL, Bosnic-Anticevich SZ, Reddel HK. Evaluation of a novel educational strategy, including inhaler-based reminder labels, to improve asthma inhaler technique. Patient Educ Couns. 2008;72(1):26-33. doi:10.1016/j.pec.2008.01.014

29. Lababidi H, Hijaoui A, Zarzour M. Validation of the Arabic version of the asthma control test. Ann Thorac Med. 2008;3(2):44-47. doi:10.4103/1817-1737.39635

30. Nathan RA, Sorkness CA, Kosinski M, et al. Development of the asthma control test: a survey for assessing asthma control. J Allergy Clin Immunol. 2004;113(1):59-65. doi:10.1016/j.jaci.2003.09.008

31. Korn S, Both J, Jung M, Hubner M, Taube C, Buhl R. Prospective evaluation of current asthma control using ACQ and ACT compared with GINA criteria. Ann Allergy Asthma Immunol. 2011;107(6):474479. doi:10.1016/j.anai.2011.09.001

32. Reddel HK, Taylor DR, Bateman ED, et al. An official American Thoracic Society/European Respiratory Society statement: asthma control and exacerbations: standardizing endpoints for clinical asthma trials and clinical practice. Am J Respir Crit Care Med. 2009;180(1):59-99. doi:10.1164/rccm.200801-060ST

33. Schatz M, Kosinski M, Yarlas AS, Hanlon J, Watson ME, Jhingran P. The minimally important difference of the Asthma Control Test. $J$ Allergy Clin Immunol. 2009;124(4):719-723.e711. doi:10.1016/j. jaci.2009.06.053

34. Basheti IA, Bosnic-Anticevich SZ, Armour CL, Reddel HK. Checklists for powder inhaler technique: a review and recommendations. Respir Care. 2014;59(7):1140-1154. doi:10.4187/respcare.02342

35. Greenway-Crombie A, Conners A, Snell T, Oerlemans M. Development of a Rural Asthma Management Model, RAMM. Rural Remote Health. 2003;3(2):149.

36. Lum EY, Sharpe HM, Nilsson C, et al. Urban and rural differences in the management of asthma amongst primary care physicians in Alberta. Can J Clin Pharmacol. 2007;14(3):e275-e282.
37. Fuhlbrigge AL, Kitch BT, Paltiel AD, et al. FEV(1) is associated with risk of asthma attacks in a pediatric population. $J$ Allergy Clin Immunol. 2001;107(1):61-67. doi:10.1067/mai.2001.111590

38. Knapp KK, Paavola FG, Maine LL, Sorofman B, Politzer RM. Availability of primary care providers and pharmacists in the United States. J Am Pharm Assoc (Wash). 1999;39(2):127-135.

39. Sulaiman I, Seheult J, MacHale E, et al. Irregular and ineffective: a quantitative observational study of the time and technique of inhaler use. J Allergy Clin Immunol Pract. 2016;4(5):900-909.e902. doi:10.1016/j.jaip.2016.1007.1009

40. Fathima M, Naik-Panvelkar P, Saini B, Armour CL. The role of community pharmacists in screening and subsequent management of chronic respiratory diseases: a systematic review. Pharm Pract (Granada). 2013;11(4):228-245.

41. Westerik JA, Carter V, Chrystyn H, et al. Characteristics of patients making serious inhaler errors with a dry powder inhaler and association with asthma-related events in a primary care setting. J Asthma. 2016;53(3):321-329. doi:10.3109/02770903. 02772015.01099160

42. Azzi E, Srour P, Armour C, Rand C, Bosnic-Anticevich S. Practice makes perfect: self-reported adherence a positive marker of inhaler technique maintenance. NPJ Prim Care Respir Med. 2017;27(1):29. doi:10.1038/s41533-41017-40031-41530

43. Shealy KM, Paradiso VC, Slimmer ML, Campbell DL, Threatt TB. Evaluation of the prevalence and effectiveness of education on metered-dose inhaler technique. Respir Care. 2017;62(7):882-887. doi: $10.4187 /$ respcare.05246

44. Schatz M, Sorkness CA, Li JT, et al. Asthma Control Test: reliability, validity, and responsiveness in patients not previously followed by asthma specialists. J Allergy Clin Immunol. 2006;117(3):549-556. doi:10.1016/j.jaci.2006.01.011

45. Ozoh OB, Okubadejo NU, Chukwu CC, Bandele EO, Irusen EM. The $\mathrm{ACT}$ and the ATAQ are useful surrogates for asthma control in resourcepoor countries with inadequate spirometric facilities. J Asthma. 2012;49 (10):1086-1091. doi:10.3109/02770903.2012.729632

46. Basheti IA, Hammad S, Alsaraj N, Reddel H. Learning and teaching inhaler technique by health care professionals: an interprofessional approach revealing needs and barriers. Jor $J$ App Sci. 2016;13:1-16.

47. Pinto Pereira L, Clement Y, Simeon D. Educational intervention for correct pressurised metered dose inhaler technique in Trinidadian patients with asthma. Patient Educ Couns. 2001;42(1):91-97.

48. Kamps AW, Brand PL, Roorda RJ. Determinants of correct inhalation technique in children attending a hospital-based asthma clinic. Acta Paediatr. 2002;91(2):159-163.

49. Basheti IA, Obeidat NM, Reddel HK. Inhaler technique education and asthma control among patients hospitalized for asthma in Jordan. Saudi Pharm J. 2018;26(8):1127-1136. doi:10.1016/j.jsps.2018. 1106.1002

\section{Publish your work in this journal}

Clinical Pharmacology: Advances and Applications is an international, peer-reviewed, open access journal publishing original research, reports, reviews and commentaries on all areas of drug experience in humans. The manuscript management system is completely online and includes a very quick and fair peer-review system, which is all easy to use. Visit http://www.dovepress.com/testimonials.php to read real quotes from published authors. 